\title{
A monolithic unified approach for FSI and multi-fluid flow problems using the Particle Finite Element Method with fixed mesh
}

\author{
P. Becker ${ }^{1}$, S.R Idelsohn ${ }^{1,2}$, E. Oñate ${ }^{1,3}$
}

Received: date / Accepted: date

\begin{abstract}
This paper describes a strategy to solve multifluid and Fluid Structure Interaction (FSI) problems using Lagrangian particles combined with a fixed Finite Element (FE) mesh . The method is an extension from the fluid-only PFEM-2 [14][15], which uses explicit integration over the streamlines to improve accuracy. As a result, the convective term does not appear in the set of equations solved in the fixed mesh. Enrichments in the pressure and field are used to improve the description of the interface between phases.
\end{abstract}

Keywords Multifluids · FSI · Fixed mesh · Lagrangian particles · Unified approach

\section{Introduction}

The simulation of Fluid Structure Interaction (FSI) problems is an area of growing interest due to the several application fields in which it is required. Nowadays all structures, from buildings to airplanes and boats are optimized to minimize costs, leading to a more flexible behaviour in air or water. Furthermore, new areas are always being explored, being biomedical research a fast growing and stimulating field.

Correspondence to: Pablo Becker

CIMNE, Edificio C1, Campus Nord UPC,

Carrer Gran Capità s/n, 08034, Barcelona, Spain

Tel.: +34934016038

Fax: +34934016 517

E-mail: pbecker@cimne.upc.edu

${ }^{1}$ Centre Internacional de Mètodes Numèrics en Enginyieria (CIMNE)

${ }^{2}$ Institució Catalana de Recerca i Estudis Avanats (ICREA)

${ }^{3}$ Departament de Resistència de Materials i Estructures a l'Engenyeria (RMEE), Universitat Politècnica de Catalunya $(U P C)$
Leaving aside one-way FSI methods, the staggered or weakly coupled algorithm is the most common approach to treat FSI problems. This method consists on computing separately the fluid and the solid domain, without ensuring force balance at each time step. The popularity of this method lies in its speed and possibility to use specialized solvers for the structure and fluid, a desired property since both subdomains are generally treated in different frameworks. While for the structural solver most algorithms employ a Lagrangian reference framework, for the fluid either Eulerian or Arbitrary Lagrangian Eulerian (ALE) frameworks are normally used.

On the other hand, in strongly coupled algorithms the solution is considered converged only when force balance is achieved at the interfaces. To couple the fluid and structural subdomains, the so called monolithic and partitioned strategies have been developed. While the first one consists on assembling and solving all the equations in a single system, the partitioned algorithm splits the fluid and solid domain, relying on an iterative process to achieve convergence.

In [16] a strongly coupled monolithic strategy is proposed to treat simultaneously the fluid and solids within the same framework. Using a Lagrangian approach, it was possible to write the momentum equations for both solids and fluids together and seamlessly with only minor differences due to the stress memory of solids. This allowed to solve al the equations within a unified computational frame, highly improving the accuracy. On the other hand, to deal with the large deformations experimented by the fluid phase, a remeshing algorithm was implemented. This allowed to avoid distorted meshes, but with the extra computational cost associated to the Delaunay triangulation meshing stage. 
Following the same line, in this work we propose a new method which uses the same discretization and solving technique for all the phases present in the domain. This strategy is an extension of the Particle Finite Element Method-second generation (PFEM-2) developed for multifluids in [15]. The PFEM-2 is based on the use of Lagrangian particles with no connectivities to convect all the material properties combined with a fixed mesh, leading to a Lagrangian formulation without the need of deforming the spatial mesh nor remeshing.

Since all the material points of the domain are represented using lagrangian particles, it is possible to convect an unlimited number of variables at almost no cost. Furthermore, the convection stage is greatly improved by following the streamlines of the velocity in an explicit way, therefore improving accuracy over first order explicit methods but keeping the computational costs low. Once the particles have been convected, the variables are projected into the mesh, boundaries are identified where material properties change and the lagrangian system is solved in the fixed mesh. Furthermore, by improving the definition of the interface using enrichment shape functions, the physics of the problems are exactly simulated despite that the mesh does not match the interfaces.

This paper is structured as follows. First, the monolithic strategy of the PFEM-2 is described, in the next sections the strategy for the treatment of the FSI problem is described and finally validation examples are presented.

\section{Monolithic strategy for multi-fluids and FSI}

\subsection{Mixed Framework: The PFEM-2}

A starting point to reduce the complexity of the problem is selecting the right reference framework. An appropriate choice leads to the simplification of complex terms and higher accuracy in the results due to better approximations. Following this line, the Lagrangian framework offers important advantages due to the simplicity of the equations. However, since material points move and the configuration changes continuously in time, it is necessary to couple this set of equations with a strategy that solves for the movement of the material points.

The current model is based on a mixed EulerianLagrangian framework, combining advantages of both methods. This is achieved by using a set of particles combined with a fixed FEM mesh in a solving strategy known as PFEM-2 [14]. The method is obtained by writing, for a given particle $p$, the following definition for the position and velocity in a given timestep $n+1$, where $\mathbf{V}$ is the velocity and $\mathbf{a}$ is the acceleration.

$$
\begin{aligned}
& \mathbf{x}_{p}^{n+1}=\mathbf{x}_{p}^{n}+\int_{n}^{n+1} \mathbf{V}^{\mathbf{t}} d t \\
& \mathbf{V}_{p}^{n+1}=\mathbf{V}_{p}^{n}+\int_{n}^{n+1} \mathbf{a}^{\mathbf{t}} d t
\end{aligned}
$$

By using an explicit strategy in (1a), the convective term is completely uncoupled from the momentum equations and Lagrangian equations are obtained. The main advantage of using the Lagrangian particles is that the convection is obtained by simply moving the particles across the space and therefore the system to be solved does not include the convective term. This set of equations is calculated on the mesh, although it is possible to include partial contribution from the particles to improve the accuracy [14]. Then the complete

\begin{tabular}{|c|c|c|c|c|}
\hline $\begin{array}{l}\text { Particle } \\
\text { Stage } \\
\text { Convect particles }\end{array}$ & $\begin{array}{l}\text { Project } \\
\text { info }\end{array}$ & & \begin{tabular}{|l|} 
Mesh \\
Stage \\
Solve eqs
\end{tabular} & $\rightarrow \begin{array}{l}\mathrm{Up} \\
\mathrm{par}\end{array}$ \\
\hline
\end{tabular}
scheme required to solve a step becomes:

The particles used in the scheme do not represent a fixed amount of mass, but rather material points with certain properties and velocity. This allows for different amount of particles to be used depending on the zone to ensure a better accuracy on those areas. It must be noted that in the algorithm presented in this paper, the particles are only used to transport the information (solve the convective term). However, in certain cases where the viscosity is low and there is a single fluid, it is possible to solve partially the momentum equation (1b) in the particles, as explained in [14]. Despite this strategy leads to higher accuracy in the cases analysed in the article, however it lacks the generality that is required for the simulation of FSI problems.

Figure 1 shows the streamline integration for a single particle. This step is purely explicit, which is achieved by convecting the particles using the velocity from the previous time step, in a Picard [9] iteration fashion. As for the force integration (if added), information is also gathered from the last step, so the Lagrangian particle step remains purely explicit, allowing for a fast computation on each particle that is trivially parallelizable and hence fast.

Remark: It is possible to imagine this explicit convection as the first step of a non linear iteration process. If an iterative process would be to follow, once the new velocity has been calculated, each particle should have its coordinates resetted to the initial position and the streamline integration will be performed again to repeat all the tasks. However, practical experience suggests that the first iteration provides results that are 


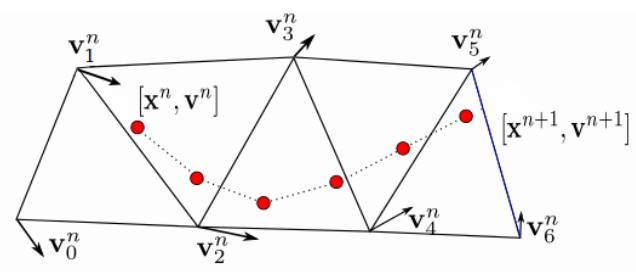

Fig. 1: PFEM-2 streamline integration

accurate enough, since numerical data matches the experimental results even with very large time steps.

Once all the particles have been convected, information is projected into the mesh. The interface is drawn by determining the exact place where the properties change. This is defined in the mesh by an auxiliary scalar function $\varphi$ that is valued zero in theses lines(2D) or surfaces $(3 \mathrm{D})$. A correct location of the interface is critical, since the finite element mesh is enriched at the interface as explained later, which allows for a more accurate solution. Figure 2 shows the position of the interface for a given distribution of particles. The projection kernel to transfer the information from the particles to the mesh is a critical part of the strategy. Since no information is stored in the mesh, this kernel must be accurate enough as to guarantee that the projected field of the variables(in the mesh) resembles the field of the original domain (particles). Failing to do so would mean that a different problem is being solved at each domain and therefore accuracy is lost.

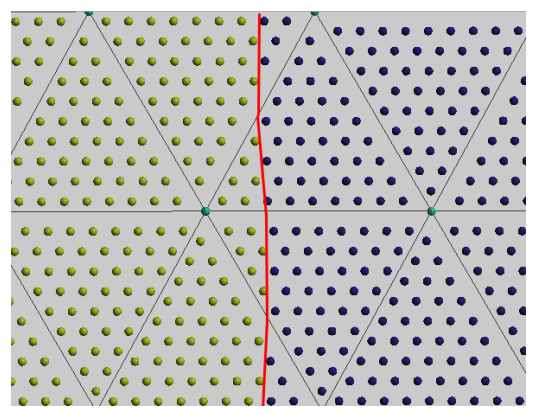

Fig. 2: Interface detection using the particles. At the red line $\varphi=0$

Having detected the interface, the system of equation is assembled and solved implicitly. Since the sound speed is infinite in incompressible fluids, the complete set of equations cannot be calculated explicitly [17].

Finally, once the full system of equations has been solved, corrections are passed to the particles and the cycle is restarted. Details of the convection stage and the procedure for solving the system of equations will be explained in the following sections.

\subsection{Continuum equations}

As explained in the last section, the use of particles allows to omit the convective term. The system of equation to be solved is obtained by coupling the momentum balance for a general material with a compressibility equation. Together, these two equations provide exact solutions for problems at which thermal terms are considered uncoupled from the mechanical equations.

$$
\begin{array}{r}
\rho \frac{D(\mathbf{V})}{D t}=\nabla \boldsymbol{\sigma}+\rho \mathbf{g} \\
\frac{D p}{D t}+\kappa \nabla \cdot \mathbf{V}=0
\end{array}
$$

Equations (2) hold for any material, both fluids and solids, where $\rho$ is the density, $\kappa$ the compressibility modulus, $\mathbf{V}$ the velocity, $\boldsymbol{\sigma}$ the stresses and $\mathbf{g}$ the mass forces.

\subsubsection{Continuum equations for fluids}

For the particular case of fluids, the stress tensor depends only on the current strain rate and the pressure: $\boldsymbol{\sigma}_{f}=2 \mu\left(\nabla \mathbf{V}^{S}\right)-\nabla p$. Since the aim of this work is to deal with problems of relatively low velocities $\|\mathbf{V}\|<0.3 c$ (where $c$ is speed of sound), the fluid will be considered incompressible. Replacing into (2), the equations for fluids become (3).

$$
\begin{array}{r}
\rho \frac{D(\mathbf{V})}{D t}=\nabla\left[2 \mu\left(\frac{\nabla \mathbf{V}+(\nabla \mathbf{V})^{T}}{2}\right)\right]-\nabla p+\rho \mathbf{g} \\
\nabla \cdot(\mathbf{V})=0
\end{array}
$$

It must be noted that this set of equations must be stabilized due to the infinite sound speed caused by the incompressibility condition.

\subsubsection{Continuum equations for solids}

The hypoelastic model provides an excellent constitutive model for solids due to its simplicity and direct application for velocity formulations. Hypoelastic materials are those whose stress can be defined simply using the rate of deformation as:

$$
\dot{\boldsymbol{\sigma}}=f(\boldsymbol{\sigma}, \mathbf{d}) \quad \text { where } \quad d_{i j}=\frac{1}{2}\left(\frac{\partial v_{i}}{\partial x_{j}}+\frac{\partial v_{j}}{\partial x_{i}}\right)
$$

The definition of hypoelastic materials return the rate of stress rather than the stress itself. This is particularly useful in the velocity formulation since the rate stress function $f$ depends on the rate of deformation d (related to the velocity) instead of the deformation. However, the Cauchy stress tensor is not objective (it is affected by rigid body rotations). Therefore, another 
stress measure is required. Using the Truesdell stress rate $\boldsymbol{\sigma}^{\nabla}$ and eliminating the stress dependency of the model for simplicity, the stress rate becomes:

$\boldsymbol{\sigma}^{\nabla}=f(\mathbf{d})$

The Truesdell rate is related to the PK2 Stress $\mathbf{S}$ and provides the exact stress rate for a given deformation.

$\boldsymbol{\sigma}^{\nabla}=J^{-1} \mathbf{F}^{(n+1)} \dot{\mathbf{S}}^{(n+1)} \mathbf{F}^{T(n+1)}$

Using the tensor of elastic moduli for the Truesdell stress rate $\mathbf{C}^{T}$ :

$$
\boldsymbol{\sigma}^{\nabla}=\mathbf{C}^{T}: \mathbf{d}
$$

$\boldsymbol{\sigma}^{\nabla}=(\lambda \mathbf{I} \otimes \mathbf{I}+2 \mu \mathbf{I}): \mathbf{d}-\mathbf{d} \cdot \boldsymbol{\sigma}-\boldsymbol{\sigma} \cdot \mathbf{d}+\boldsymbol{\sigma} \otimes \mathbf{I}$

Where $\lambda$ and $\mu$ are the Lamè parameters. As it can be seen in equation (7) it is not linear since $C$ depends on $\sigma$. To override this inconvenience, the Jaumann [1] stress rate is used, which simplifies the stress rate into a deformation $\sigma^{\nabla J}$ plus a rotation, avoiding all the nonlinear terms.

$\boldsymbol{\sigma}^{\nabla J}=(\lambda \mathbf{I} \otimes \mathbf{I}+2 \mu \mathbf{I}): \mathbf{d}$

Using the approximation of the stress rate (9) instead of the exact Truesdell stress, equation 6 can be written as:

$J^{(n+1)}(\lambda \mathbf{I} \otimes \mathbf{I}+2 \mu \mathbf{I}): \mathbf{d}^{n+1}=\mathbf{F}^{(n+1)} \dot{\mathbf{S}}^{(n+1)} \mathbf{F}^{T(n+1)}$

We use finite differences in time to obtain $\dot{\mathbf{S}}$

$$
\begin{gathered}
J^{(n+1)}(\lambda \mathbf{I} \otimes \mathbf{I}+2 \mu \mathbf{I}): \mathbf{d}^{n+1}= \\
\mathbf{F}^{(n+1)} \frac{\mathbf{S}^{(n+1)}-\mathbf{S}^{(n)}}{\Delta t} \mathbf{F}^{T(n+1)} \\
\mathbf{F}^{(n+1)} \frac{\mathbf{S}^{(n+1)}}{\Delta t} \mathbf{F}^{T(n+1)}=J^{(n+1)}(\lambda \mathbf{I} \otimes \mathbf{I}+2 \mu \mathbf{I}): \mathbf{d}^{n+1}+ \\
\mathbf{F}^{(n+1)} \frac{\mathbf{S}^{(n)}}{\Delta t} \mathbf{F}^{T(n+1)}
\end{gathered}
$$

Recalling the relation between the second PK stress and the Cauchy stress $J \sigma=\mathbf{F S F}^{T}$, the LHS becomes:

$$
\begin{array}{r}
J^{(n+1)} \frac{\boldsymbol{\sigma}^{(n+1)}}{\Delta t}=J^{(n+1)}(\lambda \mathbf{I} \otimes \mathbf{I}+2 \mu \mathbf{I}): \mathbf{d}^{n+1}+ \\
\mathbf{F}^{(n+1)} \frac{\mathbf{S}^{(n)}}{\Delta t} \mathbf{F}^{T(n+1)}
\end{array}
$$

Resetting the reference framework at each time step as the last known configuration $\mathbf{x}^{(n+1)}:=\mathbf{X}^{(n)}$, the Cauchy stress of the previous time step directly becomes the PK2 stress $\mathbf{S}^{(n)}:=\boldsymbol{\sigma}^{(n)}$. Finally the stress in the new configuration becomes (13), where the subindex $n+1$ implies that the old stresses have been updated to the new configuration.

$$
\begin{gathered}
\boldsymbol{\sigma}^{(n+1)}=J^{-1(n+1)} \mathbf{F}^{(n+1)} \boldsymbol{\sigma}^{(n)} \mathbf{F}^{T(n+1)}+ \\
\Delta t(\lambda \mathbf{I} \otimes \mathbf{I}+2 \mu \mathbf{I}): \mathbf{d}^{n+1}
\end{gathered}
$$

$\boldsymbol{\sigma}^{(n+1)}=\boldsymbol{\sigma}_{n+1}^{(n)}+\Delta t\left(\lambda \operatorname{tr}\left(\mathbf{d}^{n+1}\right) \mathbf{I}+2 \mu \mathbf{d}^{n+1}\right)$

To mimic the formulation of velocities and pressure used in fluids, it is useful to decompose the stresses into the pressure $p=-\frac{1}{3} \operatorname{tr}(\boldsymbol{\sigma})$ caused by the volumetric deformation $\epsilon_{v}=\frac{1}{3} \operatorname{tr}(\mathbf{D})$ and deviatoric part $\sigma^{\prime}$ caused by the deviatoric stress $\mathbf{d}^{\prime}=\mathbf{d}-\epsilon_{v}$.

$\boldsymbol{\sigma}^{(n+1)}=\boldsymbol{\sigma}_{n+1}^{(n)}+\Delta t\left[\lambda \operatorname{tr}\left(\mathbf{d}^{n+1}\right) \mathbf{I}+2 \mu\left(\mathbf{d}^{\prime n+1}+\epsilon_{v}\right)\right]$

$\boldsymbol{\sigma}^{(n+1)}=\boldsymbol{\sigma}_{n+1}^{(n)}+\Delta t\left[\left(\lambda+\frac{2}{3} \mu\right) \operatorname{tr}\left(\mathbf{d}^{n+1}\right) \mathbf{I}+2 \mu\left(\mathbf{d}^{\prime n+1}\right)\right]$

$\boldsymbol{\sigma}^{(n+1)}=\boldsymbol{\sigma}_{n+1}^{(n)}+\Delta p \mathbf{I}+\Delta \sigma^{\prime}$

where

$\Delta p=\Delta t\left(\lambda+\frac{2}{3} \mu\right) \operatorname{tr}\left(\mathbf{d}^{n+1}\right)$

$\Delta \boldsymbol{\sigma}^{\prime}=2 \Delta t \mu\left(\mathbf{d}^{\prime n+1}\right)$

Equations (16) and (17) provide the strategy to update the stresses at each time step. A more detailed explanation of the derivation can be found in [16] and [18].

\section{Fixed mesh domain}

\subsection{Spatial discretization}

The chosen spatial discretization is the Finite Element Method [23]. It consists on dividing the domain into elements, whose geometry is defined by nodes. The unknowns variables are the values at the nodes and the solution is interpolated from the nodal values inside each element. In this work linear shape functions will be used for all the variables.

Using the FE discretization and the test functions w for the velocity and $q$ for the pressure, the weak form [24] of the system (2) becomes (18). Note that the stress term is integrated by parts to avoid second derivatives on the shape functions.

$$
\begin{array}{r}
\left(\mathbf{w}, \rho \frac{D(\mathbf{V})}{D t}\right)_{\Omega}=(\mathbf{w}, \rho \mathbf{g})_{\Omega}-(\nabla \mathbf{w}, \boldsymbol{\sigma})_{\Omega} \\
\left(q, \nabla \cdot\left(\mathbf{V}^{n+1}\right)\right)_{\Omega}=0
\end{array}
$$


In the first equation of (18) we have omitted the boundary terms that appear due to the integration by parts. These terms are the boundary traction terms that appear at the edges of all the finite elements used in the discretization: $(\nabla \mathbf{w}, \mathbf{n} \boldsymbol{\sigma})_{\Gamma}$, where $\Gamma$ are the boundaries of the elements and $\mathbf{n}$ normal vectors pointing outwards. Ommiting this term ensures that the stress continuity is guaranteed on the inter-element boundaries, where $\Gamma_{1}$ and $\Gamma_{2}$ are the boundaries of two adjacent elements.

$$
\left(\nabla \mathbf{w}, \mathbf{n}_{1} \boldsymbol{\sigma}_{1}\right)_{\Gamma_{1}}-\left(\nabla \mathbf{w}, \mathbf{n}_{1} \boldsymbol{\sigma}_{2}\right)_{\Gamma_{2}}=0
$$

Ensuring that (19) is satisfied is of particular interest in this work since the pressure field has to be discontinous between interfaces due to the different material properties. Fullfilling this requirement by ommiting this term provides a straightforward yet accurate approach, even if discontinuities occur in the pressure field.

\subsection{Stabilized matrix form for the fluid phase}

A finite element discretization directly implemented in the form (18) using the same shape functions for the velocity and the pressure would be unstable for fluids in the pressure since it does not stand the inf-sup condition [6]. Moreover, even if different functions were to be used to avoid this restriction, the system would not be suitable for several solvers since it contains zeros in the diagonal terms of the pressure equation as seen in the matrix form (20). In these equations, first order finite differences are used in the acceleration term to advance in time.

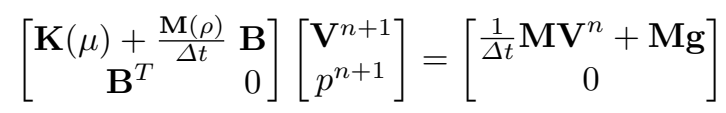

In order to raise the limitation of the original problem, stabilization terms can be added to the set of equations (18). As stated before, it is necessary to stabilize the pressure equation for the fluid problem only. While there are several methods to do so, only two will be mentioned in this work: the Pressure-Stabilizing/PetrovGalerkin (PSPG) method [11] and the Orthogonal Subgrid Scale (OSS) stabilization [4]. The reason behind this is that both methods only add a laplacian on the LHS, holding the symmetry of the system.

In (21) the final shape of the matrix system is presented for the PSPG method. Using the OSS method with an explicit projection stage would lead to a similar expression but with the projecion of the pressure in the RHS.

$$
\left[\begin{array}{cc}
\mathbf{K}(\mu)+\frac{1}{\Delta t} \mathbf{M}(\rho) & \mathbf{B} \\
\mathbf{B}^{T} & \tau L\left(\frac{1}{\rho}\right)
\end{array}\right]\left[\begin{array}{l}
\mathbf{V}^{n+1} \\
p^{n+1}
\end{array}\right]=\left[\begin{array}{c}
\frac{1}{\Delta t} \mathbf{M} \mathbf{V}^{n}+\mathbf{M g} \\
\tau \mathbf{B}^{T} \mathbf{g}
\end{array}\right]
$$

where $\mathbf{L}\left(\frac{1}{\rho}\right)$ is the laplacian of the pressure. An enhanced stabilization procedure based on the Finite Increment Calculus (FIC) avoids the need to presuppose the pressure in free surface solvers using staggered schemes. An extended updated mixed finite element lagrangian formulation for treating both quasi and fully incompressible fluids as well as FSI problems is presented in [19].

The system (21) can be solved as it is by choosing an appropriate stabilization parameter $\tau$. In this work it is set to $\tau=\left(1 / \Delta_{t}+4 \mu / h^{2}+2 V^{n} / h\right)^{-1}$ following the work by [5]. The dependency on $\Delta t$ was added to avoid $\tau \rightarrow \infty$ when the velocity and the viscosity are zero, following the work of [2].

\subsection{Matrix form for the solid phase}

Unlike fluids, solids will not be considered incompressible. The spatial discretization and variables will be the same as the one used in the fluids formulation: linear elements for both the pressure and the velocity (3 noded triangles in $2 \mathrm{D}$ and 4 noded tetrahedrons in $3 \mathrm{D}$ ). As for the deviatoric stress, it will not be an independent variable, but a state variable depending on the velocity unknowns.

Recalling the general system of equations (2) and splitting the stresses into its deviatoric and volumetric components, the system reads:

$$
\begin{array}{r}
\rho\left(\frac{D(\mathbf{V})}{D t}\right)=\nabla\left(\boldsymbol{\sigma}^{\prime}-p \mathbf{I}\right)+\rho g \\
\frac{D p}{D t}+\kappa \nabla \cdot \mathbf{V}=0
\end{array}
$$

Writing the weak form of the problem and integrating by parts the gradient of the stresses in order to avoid second derivatives on the velocities and to convert the gradient of the pressure into the transpose of the divergence, the system becomes:

$$
\begin{array}{r}
\left(\mathbf{w}, \rho \frac{D(\mathbf{V})}{D t}\right)=-\left(\nabla \mathbf{w},\left(\boldsymbol{\sigma}^{\prime}-p \mathbf{I}\right)\right)+(\mathbf{w}, \rho \mathbf{g}) \\
\left(q, \frac{D p}{D t}+\kappa \nabla \cdot \mathbf{V}\right)=0
\end{array}
$$

A first approximation in time for the velocity would lead to an stable but too diffusive algorithm. To avoid this shortcoming, the Newmark's Beta method was selected. Parameters were set for constant acceleration. 
The resulting expression for the velocity integration becomes:

$$
2 \rho \frac{\mathbf{V}^{n+1}}{\Delta t}=2 \rho \frac{\mathbf{V}^{n}}{\Delta t}-\rho \dot{\mathbf{V}}^{n}
$$

Using finite differences in time for the pressure, dividing the second equation by the compressibility modulus $\kappa$ and recalling the definition of the stresses (16) gives:

$$
\begin{aligned}
&\left(\mathbf{w}, \rho \frac{\mathbf{V}^{n+1}}{\Delta t}\right)-\left(\nabla \mathbf{w},\left(\Delta \boldsymbol{\sigma}^{\prime}-\Delta p \mathbf{I}\right)\right)= \\
&\left(\mathbf{w}, \frac{\mathbf{V}^{n}}{\Delta t}+\rho \mathbf{g}\right)-\left(\nabla \mathbf{w},\left(\Delta \boldsymbol{\sigma}_{n+1}^{\prime n}-\left(p^{n}\right) \mathbf{I}\right)\right) \\
&\left(q, \frac{p^{n+1}}{\kappa \Delta t}+\nabla \cdot \mathbf{V}\right)=\left(q, \frac{p^{n}}{\kappa \Delta t}\right)
\end{aligned}
$$

To account for the increase in the deviatoric stresses $\Delta \sigma^{\prime}$, an implicit scheme is used to avoid instabilities:

$$
\mathbf{d}=\mathbf{S} \mathbf{V}^{n+1}
$$

where $\mathbf{S}$ is the standard strain-rate velocity matrix. Finally, writing the equations in matrix form, the system can be expressed as:

$$
\begin{gathered}
{\left[\begin{array}{cc}
\left(\frac{2}{\Delta t} \mathbf{M}(\rho)+\Delta t \mathbf{K}\right) & \mathbf{B} \\
\mathbf{B}^{T} & \frac{1}{\Delta t} \mathbf{M}\left(\frac{1}{\kappa}\right)
\end{array}\right]\left[\begin{array}{c}
\mathbf{V}^{n+1} \\
p^{n+1}
\end{array}\right]=} \\
{\left[\begin{array}{c}
\mathbf{M}(\rho)\left(\frac{2}{\Delta t} \mathbf{V}^{n}-\dot{\mathbf{V}}^{n}+\mathbf{g}\right)+\mathbf{B} \boldsymbol{\sigma}_{n+1}^{\prime n} \\
\frac{1}{\Delta t} \mathbf{M}\left(\frac{1}{\kappa}\right) p^{n}
\end{array}\right]}
\end{gathered}
$$

In general all the matrices have the same form as in the fluid elements, except for the stiffness matrix $\mathbf{K}$. Since it has to account for deviatoric stresses only, it is constructed modifying the constitutive matrix in the following way:

$$
\mathbf{K}=\int_{\Omega} \mathbf{S}^{\mathbf{T}} \mathbf{D} \mathbf{S}
$$

where $\mathbf{D}$ is the constitutive matrix. It must be noted that even for $2 \mathrm{D}$ simulations, the coefficients in $\mathbf{D}$ do not change. The only modification is the elimination of the strains in the third dimension, meaning that a plain strain state is considered in $2 \mathrm{D}$ cases.

$\mathbf{S}=\left[\begin{array}{ccc}\frac{\partial N}{\partial x} & 0 & 0 \\ 0 & \frac{\partial N}{\partial y} & 0 \\ 0 & 0 & \frac{\partial N}{\partial z} \\ \frac{\partial N}{\partial y} & \frac{\partial N}{\partial x} & 0 \\ 0 & \frac{\partial N}{\partial z} & \frac{\partial N}{\partial y} \\ \frac{\partial N}{\partial z} & 0 & \frac{\partial N}{\partial x}\end{array}\right] ; \mathbf{D}=\mu\left[\begin{array}{cccccc}4 / 3 & -2 / 3 & -2 / 3 & 0 & 0 & 0 \\ -2 / 3 & 4 / 3 & -2 / 3 & 0 & 0 & 0 \\ -2 / 3 & -2 / 3 & 4 / 3 & 0 & 0 & 0 \\ 0 & 0 & 0 & 1 & 0 & 0 \\ 0 & 0 & 0 & 0 & 1 & 0 \\ 0 & 0 & 0 & 0 & 0 & 1\end{array}\right]$

The system of equations (26) can be solved without the use of stabilized formulations as long as the solid material is not incompressible. Otherwise, the pressure mass matrix becomes zero and therefore stabilization is required. However this would not be a complication since the same simple stabilization technique used in the fluid elements could be used.

Finally an additional hypothesis has to be taken in order to linearise the system. The transformation of the stresses in the previous configuration to the new configuration requires the updated velocity $\mathbf{V}^{n+1}$, making the system non-linear. The simplification used in this work consists on using the previous step velocity, therefore assuming small accelerations for the solids. This hypothesis is similar to the one used for the convective term, which showed good accuracy in all the cases tested.

\subsection{Special considerations on the interface}

Despite the advantages that the FE discretization provides, it bears a severe limitation when the variables require abrupt changes that the chosen $\mathrm{FE}$ space is not able to reproduce. As an example,when there is a sharp change of the density in a two fluid problem, the hydrostatic condition under the gravitational force leads to two different values for the pressure gradient. This is specially critical when the fluids have very different densities (i.e. air-water), where the term $\nabla p=\rho g$ changes abruptly on the interface.

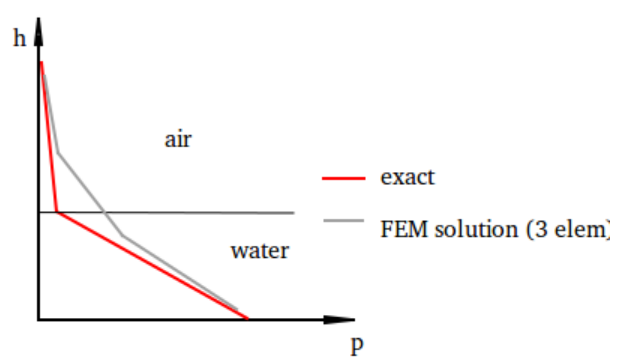

Fig. 3: Pressure distribution for the hydrostatic case

Figure 3 shows the exact pressure distribution for the hydrostatic case of water and air and the one obtained by three linear elements. It is clear that this solution is very poor and in practice it would lead to an incorrect behavior of the interface and mass losses $[7]$.

When dealing with FSI problems, this issue is even more critical. The reason for this is again the strong discontinuity in the material properties, in particular the stiffness (or viscosity). A simple example to illustrate 
this is a box of a dense solid material surrounded by air under gravitational force. In this case the pressure distribution would take the shape illustrated in Figure 4.

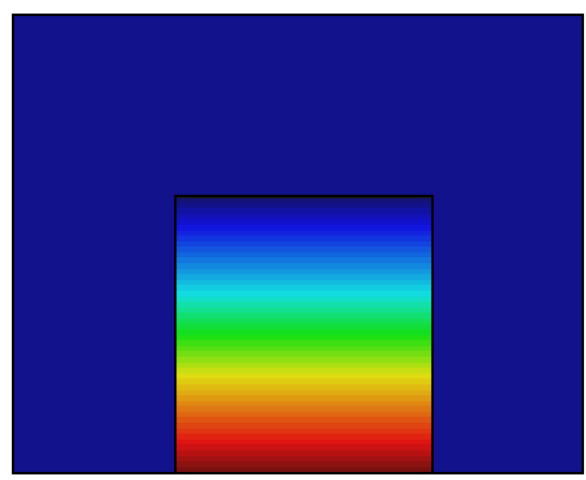

Fig. 4: Pressure distribution of a solid body surrounded by air under gravitational force

The fulfillment of the transmission conditions requires that the tractions along the interface must be continuous. Neglecting air pressure, this condition is satisfied by $\sigma_{x x}=0$ in the boundaries of the solid. However the implemented solid model makes use of the mean solid pressure, which means that (taking $\sigma_{z z}=0$ ) the pressure in the solid becomes (28):

$$
p_{\text {solid }}=\frac{1}{3} \sigma_{y y} \neq p_{\text {air }}
$$

The pressure (28) is clearly discontinuous along the interface. If a linear (or any continuous) element was located over the sharp boundary, the discrete pressure field would detect a non-existent gradient that would create normal velocities in the fluid, as seen in Figure 5. Since this would violate fluid incompressibility, the solver usually converges to a solution where the last solid node has very low pressure and therefore the structure is more flexible than it should be.

\subsection{Raising the limitations: pressure enrichments}

To overcome the problems of the standard Finite Elements, the space must be modified to allow for a more accurate reproduction of the solution. A first alternative would be remeshing the zone where the interface passes through. This would lead to the exact solution but would require adding new degrees of freedom (DoFs) in the new nodes. This strategy, despite possible, is computationally expensive since the system must be resized and new space must be allocated in the memory, task

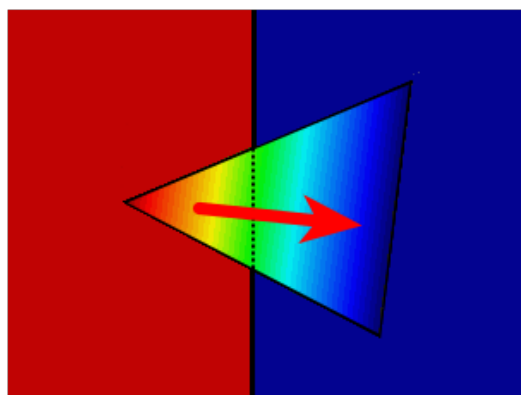

Fig. 5: Close-up of a linear element located at the interface showing the interpolated pressure field

that is likely to be slower than the solution of the system itself.

An alternative to a new mesh is enrichening the FE space. Enriching consist on creating new DoFs on each side of the interface elements and then statically condensing the new unknowns [10].

To account for the gradient discontinuity in the pressure, the shape functions proposed by [?] are used. In Figure 6 it can be seen that a total of 6 functions are used, with the objective of uncoupling completely the pressure from both sides. To do so, first the standard shape functions in the interface elements are replaced. These functions are basically the same as the original ones, but the integrals are calculated only with the contribution of the partitions whose sign matches the sign of the node. When partitions and nodes have different the same sign, the contribution is added to the enrichments functions $i^{*}, j^{*}$ or $k^{*}$. In other words, the original shape functions are split in two across the interface, allowing more freedom in the pressure field while retaining the partition of unity.
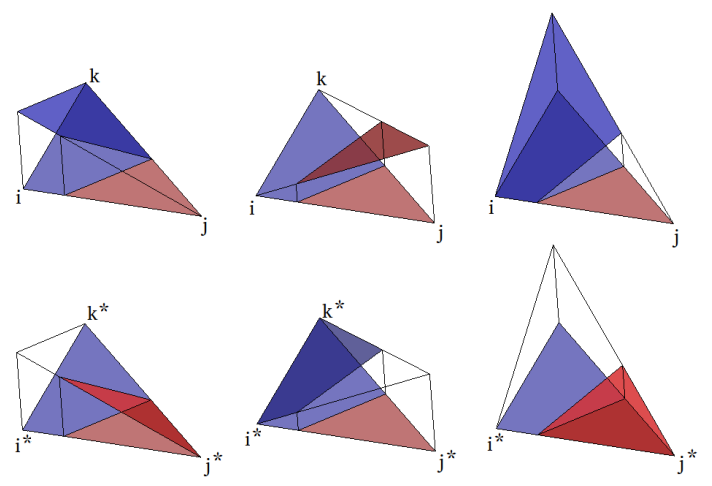

Fig. 6: Pressure Shape Functions: Top: Replacement Functions. Bottom: Enrichments 
Stabilizing the pressure equation is equivalent to adding artificial diffusion to this variable. Therefore adding stabilization to the interface elements would inevitably lead to an innacurate behavior of the solid phase due to dissipation of the stresses. For this reason no stabilization is used in these elements. However, as stated in the previous sections, doing so would cause instabilities due to the non-fulfiliment of the inf-sup [4] condition. To override this problem, both sides of the interface are considered to be compressible (using the compressibility modulus of the solid phase). While this hypothesis is not correct, it leads to small errors only since the fluid volume is changed only when there is a sharp change in the pressure from one timestep to the next. This strategy yields almost exact results, avoiding pressure diffusion across the interface with minimal errors.

Remark: One of the advantages of this set of functions is that the partition of unity is conserved. But most importantly, this also implies that reconstructing the pressure $p_{n}$ after convection is easier to perform accurately. The strategy currently implemented consists on the following steps: First, a standard nodal projection algorithm is used and, therefore, the values of the replacement shape functions are set. Having done this, the enrichments shape functions are defined as the mean of the real nodal values for the previous timestep $n$. This ensures that the prediction for the gradient in the normal direction is minimized, reducing spurious velocities.

\subsubsection{Adding the new DoFs to the system of equations}

The described enrichment functions can be added in the Monolithic strategy following the standard condensing procedure [10]. Defining the notation $N$ for the standard shape functions and $*$ for the enrichments, the extended system becomes (29). The $\rho$ is not written in the velocity mass matrices due to lack of space. Also, since all mass matrices (both velocity and pressure) are multiplied by $\frac{1}{\Delta t}$, it is also omitted for the same reason.

$$
\begin{array}{r}
{\left[\begin{array}{cc|c}
\mathbf{K}_{N N}+\mathbf{M}_{N} & \mathbf{B}_{N N} & \mathbf{B}_{N *} \\
\mathbf{B}_{N N}{ }^{T} & \mathbf{M}_{N}\left(\frac{1}{\kappa}\right) & 0 \\
\hline \mathbf{B}_{N *}{ }^{T} & 0 & \mathbf{M}_{*}\left(\frac{1}{\kappa}\right)
\end{array}\right]\left[\begin{array}{c}
\mathbf{V}_{\mathbf{N}}^{n+1} \\
p_{N}^{n+1} \\
\hline p_{*}^{n+1}
\end{array}\right]} \\
=\left[\begin{array}{c}
\frac{1}{\Delta t} \mathbf{M}_{\mathbf{N}} \mathbf{V}_{\mathbf{N}}^{n}+\mathbf{M}_{\mathbf{N}} \mathbf{g} \\
\mathbf{M}_{N}\left(\frac{1}{\kappa}\right) \mathbf{p}_{N}^{n} \\
\hline \mathbf{M}_{*}\left(\frac{1}{\kappa}\right) \mathbf{p}_{*}^{n}
\end{array}\right]
\end{array}
$$

To simplify notation, the system is written as:

$$
\left[\begin{array}{c|c}
\mathbf{A}_{\mathbf{N N}} & \mathbf{A}_{\mathbf{N} *} \\
\hline \mathbf{A}_{* \mathbf{N}} & \mathbf{A}_{* *}
\end{array}\right]\left[\frac{\mathbf{X}_{\mathbf{N}}}{\mathbf{X}_{*}}\right]=\left[\begin{array}{c}
\mathbf{F}_{\mathbf{N}} \\
\hline \mathbf{F}_{*}
\end{array}\right]
$$

Condensing the enrichment DoFs the initial size of the system is recovered, as:

$$
\tilde{\mathbf{A}} \mathbf{X}_{\mathbf{N}}=\tilde{\mathbf{F}}
$$

where

$$
\begin{aligned}
& \tilde{\mathbf{A}}=\mathbf{A}_{\mathbf{N N}}-\mathbf{A}_{\mathbf{N} *} \mathbf{A}_{* *}^{-1} \mathbf{A}_{* \mathbf{N}} \\
& \tilde{\mathbf{F}}=\mathbf{F}_{\mathbf{N}}-\mathbf{A}_{\mathbf{N} *} \mathbf{A}_{* *}^{-1} \mathbf{F}_{*} \tilde{\mathbf{F}}
\end{aligned}
$$

\section{Lagrangian particles domain}

\subsection{Streamline integration}

The solving strategy described in the previous section requires an accurate and robust tool to account for the convective term. Since the aim of the PFEM-2 is to achieve large time steps with small errors, it is not enough to simply move the particles with the last velocity multiplied by the time step $\left(\delta \mathbf{x}=\mathbf{V}^{\mathbf{n}} \cdot \delta_{\mathbf{t}}\right)$.

The main objective is to obtain as much information as possible from the previous time step to calculate the new state of the particles. The method chosen, following the same strategy of [14] for single fluids, is the streamline integration. For a particle $p$, the exact position at time $n+1$ is defined as (34):

$\mathbf{x}_{p}^{n+1}=\mathbf{x}_{p}^{n}+\int_{n}^{n+1} \mathbf{V}^{\mathbf{t}} d t$

Unfortunately, the velocity is not known at continuously in the time $t$ but only at the discrete time steps( $n-1, n, n+1 \ldots)$. Approximating now the trajectory using the velocity of the previous time step, the new position becomes:

$\mathbf{x}_{p}^{n+1} \approx \mathbf{x}_{p}^{n}+\int_{n}^{n+1} \mathbf{V}^{\mathbf{n}} d t$

While this approximation may be valid for quasistationary problems, it can lead to severe errors in transient phenomena. In [14] it was shown that for single fluids this strategy is valid. However in multi-fluids and FSI problems the difference in the properties, in particular the density, makes this approximation unsuitable for some cases. When the density jump is important, one fluid dominates over the other and their behaviour is completely different (ie: air and water). As an example, a breaking wave is represented in Figure 7. Following the streamlines to predict the water particle trajectory would lead to incorrect results.

To overcome this problem, the streamline integration must be deactivated when a particle of the heavier fluid transits across a region of the lighter fluid. In this case, the gravity will be taken as the only acting force, 


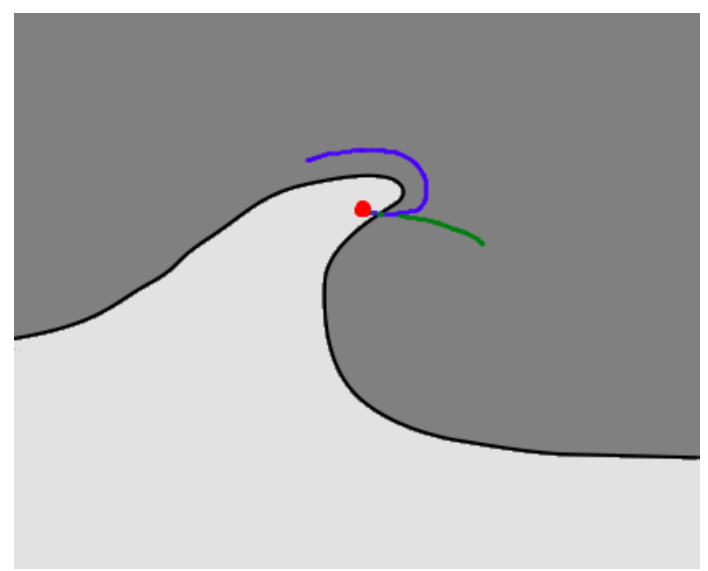

Fig. 7: Breaking wave with two fluids. Blue line: Streamline at $t^{n}$; Green line: Actual particle trajectory

leading to parabolic (projectile) motion, where the last known velocity of the particle is taken as the starting data. Setting the distance function $\varphi \leq 0$ for the denser fluid regions and $\varphi>0$ for the lighter fluid regions, the updated position of the denser fluid particles become (36):

$\mathbf{x}_{p}^{n+1} \approx\left\{\begin{array}{cl}\mathbf{x}_{p}^{n}+\int_{n}^{n+1} \mathbf{V}^{\mathbf{n}} d t \quad \text { if } \quad \varphi_{x_{p}^{t}} \leq 0 \\ \mathbf{x}_{p}^{n}+\int_{n}^{n+1} \mathbf{V}_{\mathbf{p}}^{\mathbf{n}}+g \cdot t d t & \text { if } \varphi_{x_{p}^{t}}>0\end{array}\right.$

It is important to notice that this method is purely explicit and therefore no further corrections are done for computating the convective term. Even though an iterative process could be performed to improve the accuracy, in all the examples solved with this strategy the results were correct, despite the large time steps chosen. This is specially interesting since the PFEM-2 was compared against other codes that make use of implicit strategies and yet they have to rely on smaller time steps to obtain similar results. Based on these tests it was decided to keep only the explicit computation of the convective term, since it provides an excellent balance between computation time and accuracy.

\subsection{Projection kernel}

Once the particles have been convected to their new position $\mathbf{x}_{n+1}^{p}$ it is necessary to project the information into the mesh in order to solve the Lagrangian system of equations. The simplest projection kernel consists on directly using the shape functions of the elements. As an example, the definition of the interface for two materials is explained.

Each particle has an associated material and the interface should be located where the material prop- erties change. The distribution of particles inside each element can define complicated curves that become impossible to manage with simple shape functions due to the large number of particles. As an example, in Figure 8 some particles are in the "wrong" side of the interface. For this reason the instantaneous local interface inside each element is simply defined by a line (or a plane in 3D) taking into account a weighted average using the shape functions. Assuming that only two materials are present (ie: air and water), the lighter one is given a positive sign and the denser one a negative sign. A pseudo, instantaneous, $\varphi$ level-set function is created that defines the interface where it is valued zero. To calculate the value of $\varphi$ at each the $j$ th node, all the $i$ particles in the neighbour elements to $j$ will be used. The weighting function is defined as the standard shape function of the element for the node $j$ in the position of each $i$ particle. Once the contribution of all the particles has been added, the location of the interface is trivial using (37).

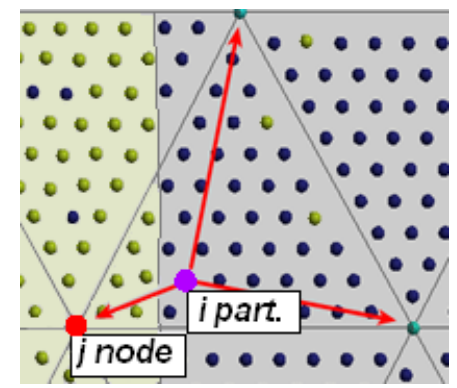

Fig. 8: Contribution of $i$ particle to the $j$ node

$\varphi_{j}=\frac{\sum_{i}^{n} \operatorname{sign}_{i} N_{i}^{j}}{\sum_{i}^{n} N_{i}^{j}}$

The same procedure is extended for all the variables, replacing the $\operatorname{sign}_{i}$ of the particle with the desired variable, either scalar, vectorial or tensorial. Using this kernel, the projected velocity can be written as (38). The $(\hat{.})$ in the notation implies that it is the first approximation of the velocity, taking into account only the convecting term and neglecting all the other contributions. This velocity will be used as the starting point for the finite element fixed mesh solver.

$\hat{\hat{\mathbf{V}}}_{j}^{n+1}=\frac{\sum_{i}^{n} \mathbf{V}_{i} N_{i}^{j}}{\sum_{i}^{n} N_{i}^{j}}$

It is worth noting that the elemental shape functions $N_{i}$ are not the only possible kernel. As an example, the function proposed in [22] assigns a higher weight to those particles that are closer to the node. Setting $h$ as 
the element size and $u=R / h$, where $R$ is the distance from the particle to the node, the Wendland kernel is written as (39).

$W_{i}^{j}=\frac{7}{4 \pi h^{2}}(1+2 u)(1-u / 2)^{4}$;

After the information of the Lagrangian domain has been projected and the mesh computations have been performed, the velocity of the particles has to be updated. At this stage it is important not to replace the particle velocity with the new, updated velocity but only a correction. The reason for this is simple; no matter how good the kernel algorithm may be, it is never exact. Replacing the unknowns in the particles would destroy valuable data, leading to excessive artificial diffusion of the strategy. Based on this, the update stage on the particle will only transfer the variation in the velocity (or any other variable) calculated in the mesh, that is:

$\delta \mathbf{V}_{j}^{n+1}=\mathbf{V}_{j}^{n+1}-\hat{\hat{\mathbf{V}}}_{j}^{n+1}$

\subsection{Computational issues and code speed}

The code used in this work was developed in the $\mathrm{C}++$ programming environment KRATOS [8]. KRATOS is an open source multi-physics framework specifically designed for finite elements, which eases the tasks that are common to all FE codes, such as the assembly of the system of equations or finding an efficient solver. Ensuring low execution times at the particles convection stage is critical since the number of particles is 10 to 20 times larger than the number of elements in the mesh for a given domain, meaning that millions of particles have to be displaced at each time step. The optimization and Parallelization of this stage lead to a code that spends approximately half of the execution time on particle tasks and the other half on the solution of the implicit system of equations in the mesh.

The current implementation is capable of solving two phase flows accurately and with execution times similar or even faster than industry standard codes such as OpenFoam, as shown in [14] for one phase flows and in [15] for multi-fluids.

\section{Complete PFEM-2 algorithm}

Coupling the strategy described in the previous sections, the PFEM-2 solver for multi-fluids and FSI is obtained. The steps can be summarized as follows:
Step 1) Convect the particles using the streamlines

Step 2) Project information on the mesh, with $q$ fluid particles and $r$ solid particles

$$
\begin{aligned}
& \text { Common variables } \\
& p_{j}^{n}=\frac{\sum_{i}^{r} p_{i} N_{i}^{j}}{\sum_{i}^{r} N_{i}^{j}} \\
& \nu_{\text {solid }}=\frac{\sum_{i}^{r} n u_{i}}{\sum_{i}^{r} 1} \\
& \mu_{\text {fluid }}=\frac{\sum_{i}^{q} \mu_{i}}{\sum_{i}^{q} 1} \\
& \boldsymbol{\sigma}_{\text {solid }}^{\prime n}=\frac{\sum_{i}^{r} \boldsymbol{\sigma}_{i}^{\prime n}}{\sum_{i}^{r} 1} \\
& \rho_{\text {fluid }}=\frac{\sum_{i}^{q} \rho_{i}}{\sum_{i}^{q} 1} \\
& E_{\text {solid }}=\frac{\sum_{i}^{r} E_{i}}{\sum_{i}^{r} 1} \\
& \rho_{\text {solid }}=\frac{\sum_{i}^{r} \rho_{i}}{\sum_{i}^{r} 1}
\end{aligned}
$$

Step 3) Detect interface elements

Step 4) Estimate pressure enrichments

Step 5) Assemble system of equations with enrichments

Step 6) Solve the system of equations

Step 7) Recover condensed DoFs

Step 8) Update the velocity at the particles, pressure and stress

\section{Numerical Examples}

\subsection{Rayleigh-Taylor instability}

The classical benchmark for multi-fluids was tested to verify the solver. Despite there are no analytical results or experimental data in $2 \mathrm{D}$ to check the error, it is possible to compare against other numerical results. $\mathrm{He}$ et al. [13] presented results for Reynolds number $R e=$ 256 using a Lattice Boltzmann scheme. With the same initial conditions, results for $R e=1000$ can be found in [12]. The geometry can be seen Figure 9, discretized by 163000 elements. The parameters of the simulation are:

$$
\begin{aligned}
& \rho_{q}=3 \mathrm{~kg} / \mathrm{m}^{3} \quad \nu_{q}=\frac{\sqrt{g} \cdot d^{3 / 2}}{R e} \mathrm{~m}^{2} / \mathrm{s} \\
& \rho_{p}=1 \mathrm{~kg} / \mathrm{m}^{3} \quad \nu_{p}=\frac{\sqrt{g} \cdot d^{3 / 2}}{R e} \mathrm{~m}^{2} / \mathrm{s} \\
& g_{y}=-10 \mathrm{~m} / \mathrm{s}^{2} \\
& \delta t=0.01 \mathrm{~s} \\
& \text { Initial conditions: } \\
& V=0 \mathrm{in} \Omega \\
& \varphi=-y-\delta_{0}(\cos (2 \pi(x)-\pi)+1)+2.0 \\
& \delta_{0}=0.1
\end{aligned}
$$

In Figure 11, snapshots at different time steps can be seen. For the Reynolds number analysed, the results obtained match the ones found in [13],[12]. The adimensionalized time $t_{a d i m}=t \sqrt{g / 2}$ is used. Finally the po- 


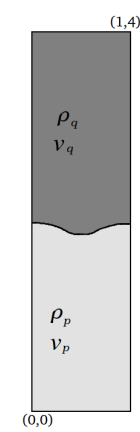

Fig. 9: Rayleigh-Taylor instability configuration

sition of the advancing front of the denser fluid (centre) and the rising of the bubbles (sides) are compared with the results of [12]. Again good matching is observed.

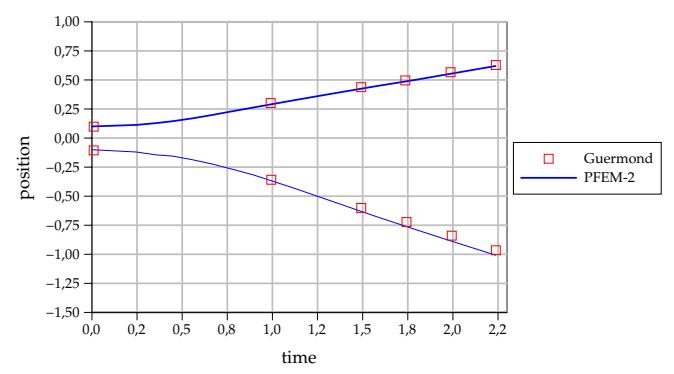

Fig. 10: Vertical position of the advancing front and bubbles in the Rayleigh-Taylor instability for $R e=1000$
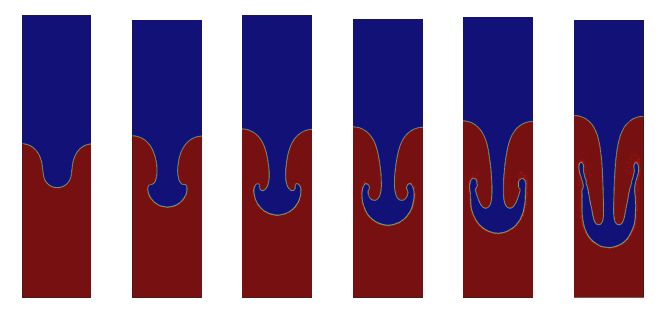

Fig. 11: Snapshots for the RayleighTaylor instability problem at $t_{\text {adim }}=$ $1.0,1.5,1.75,2.0,2.25,2.5 . R e=256$

\subsection{Static cantilever beam immersed in fluid}

To test the accuracy of the solver and the potential of the enrichment functions to uncouple the pressure, the following numerical example was designed. A beam under small deformations is surrounded by a very light fluid. Inertial terms are neglected to test the static solution, leading to a Stokes problem in the fluid. The viscosity is set as small as possible to allow for a faster convergence to the statical solution while maintaining stability. The geometry is observed in Figure 12. The material properties are:

$$
\begin{array}{ll}
\rho_{q}=1 \mathrm{~kg} / \mathrm{m}^{3} & \rho_{p}=0.001 \mathrm{~kg} / \mathrm{m}^{3} \\
E=10^{7} \mathrm{~Pa} & \mu_{p}=0.01 \mathrm{~Pa} . \mathrm{s} \\
\nu=0 &
\end{array}
$$

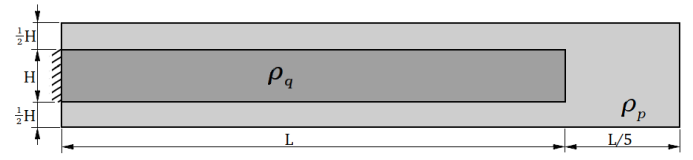

Fig. 12: Cantilever beam with fluid geometry

The beam dimensions are $L=10$ and $H=1$. A vertical mass force of $q=1\left[\mathrm{~m} / \mathrm{s}^{2}\right]$ is applied to the whole domain. According to the Timoshenko beam theory and correcting the force to account for the buoyancy effect, the vertical displacement $w$ at the tip should be

$w_{\max }=\left(\rho_{q}-\rho_{p}\right)\left(\frac{q L^{4}}{8 E I}+\frac{1}{2} \frac{q L^{2}}{10 / 12 h \mu}\right)=0.001511$

Structured meshes with decreasing element size were created to check convergence. Mesh sizes from $h=1$ to $h=0.01$ were used. To test the influence of the projection algorithm, the simulation was run with and without projection. In the latter case, the nodal pressure is kept instead of being obtained from the particles.

In order to test the behaviour of the splitted elements, in all the meshes the interface between the solid phase and the fluid phase is located at approximately the middle of the elements, as shown in Figure 13. In this case the enrichment shape functions in the pressure are imperative due to the strong discontinuity of the material properties, leading to a jump in the pressure field. If this variable was not enriched to allow for discontinuities, a strong pressure gradient would appear at the interface and, therefore, the resulting velocity field would be inaccurate. It must be noticed that Figure 13 does not correctly represent the pressure at the interface elements, since the field is printed with linear interpolation, while in reality it is discontinuous due to the enrichments.

From Figure 14 it is clear the detrimental effect of the projection stage. While the algorithm without projection is able to maintain the quadratic convergence of 


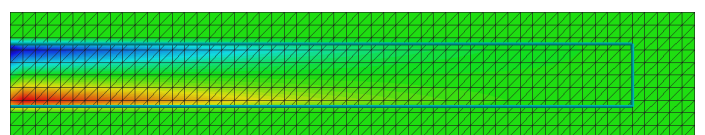

Fig. 13: Beam interface and pressure distribution

the solid-only case, the results with projection show a convergence slope of only $3 / 2$.

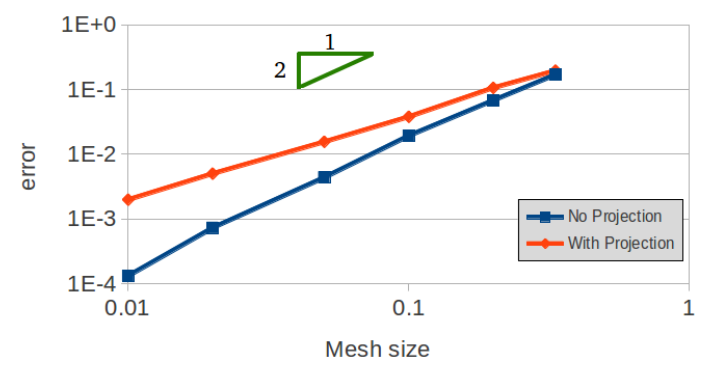

Fig. 14: Convergence for the cantilever beam in fluid

Despite the solver with the projection stage provides a less accurate solution, it leads to acceptable errors for all the mesh sizes chosen. Even for coarse meshes of only three elements along the thickness, the errors are still low (at around $10 \%$ ).

\subsection{Turek FSI benchmark}

To test the strategy in a fully coupled scenario, the Turek FSI benchmark [20] was simulated. This problem typically used as a reference to validate new FSI strategies and it is particularly challenging for staggered schemes due to the similar properties of the fluid and the solid. On the other hand, the monolithic solver used in this work does not have any particular difficulties.

The problem consists on a $2 \mathrm{D}$ rigid cylinder attached to an elastic beam immersed in a fluid current. The complete dimensions and probem parameters can be found in [20]. In this paper a fixed mesh of 48500 triangles was used. The frequency and amplitude of the oscilations are analyzed to test the accuracy. The case tested in this work is for $R e=200$, with a parabolic inlet velocity profile and the following parameters:

$$
\begin{array}{ll}
\rho_{\text {fluid }}=1000 \mathrm{~kg} / \mathrm{m}^{3} & \mu_{\text {fluid }}=1 \text { Pa.s } \\
\rho_{\text {solid }}=1000 \mathrm{~kg} / \mathrm{m}^{3} & E_{\text {solid }}=1.4 \text { MPas } \\
\nu_{\text {solid }}=0.4 & \delta t=0.005 \mathrm{~s}
\end{array}
$$

In Figure 15 the velocity contour is observed after the stationary regime has been achieved. Finally, in Fig- ure 16 the vertical displacement of the tip can be observed. In Table 1 the results are analyzed. Compared to Turek's solution, both the amplitude and frequency are $10 \%$ below the reference value. This result is considered acceptable considering that only ten triangular elements are present in the thickness of the beam.

\begin{tabular}{|l|cr|}
\hline & Frequency & Amplitude \\
\hline Turek & 5.3 & 0.0707 \\
PFEM2 & 4.8 & 0.0610 \\
\hline
\end{tabular}

Table 1: PFEM2 and Turek results

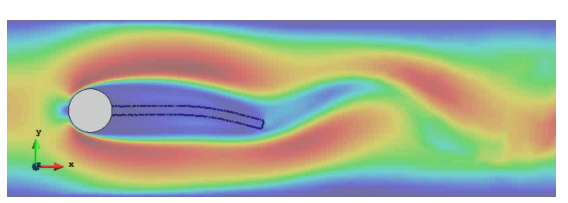

Fig. 15: Turek FSI velocity contour

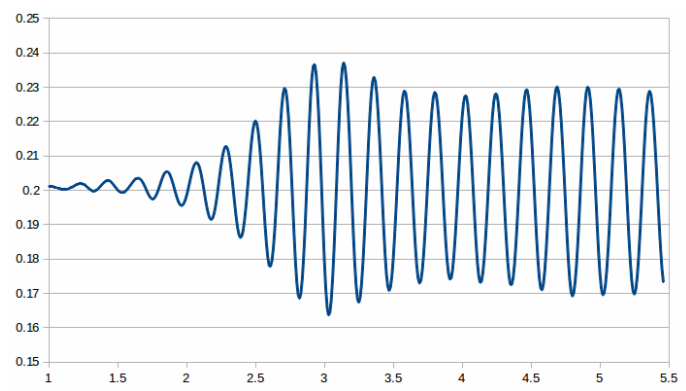

Fig. 16: Tip displacement of the Turek FSI

\subsection{D benchmark: dynamic cantilever beam}

As the last example case to test the solver in dynamic tridimensional problems, the same geometry of the previous $2 \mathrm{D}$ cantilever beam was selected but restoring the inertial terms and taking the width $b=1$ to obtain a square section. The geometry can be seen in Figure 17, with a vertical mass force to initiate the movement. Chosen mesh size is $h=0.125$ in the surroundings of the beam and $h=0.6$ in the distant fluid areas, for a total of 283500 tetrahedrons and more than 4 million particles. The time step is set to $\Delta t=0.001$ and the properties of the solid are the same as the $2 \mathrm{D}$ case:

$$
\rho=1 \mathrm{~kg} / \mathrm{m}^{3} \quad E=10^{7} \mathrm{~Pa} \quad \nu=0
$$




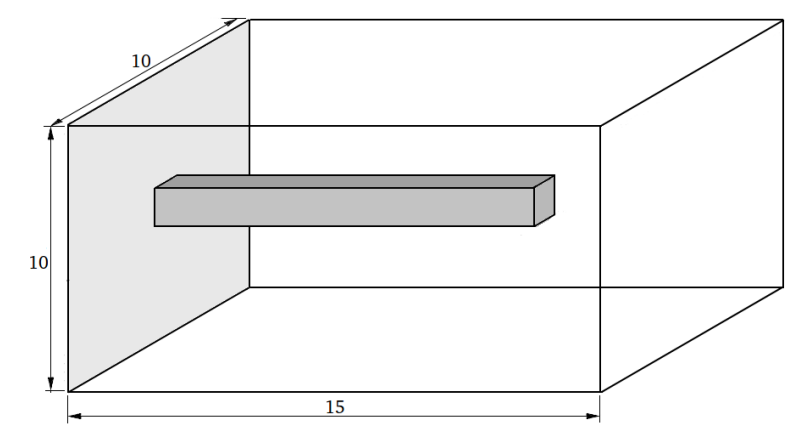

Fig. 17: Tridimensional Cantilever geometry

For cases in which the density of the fluid is negligible, it is possible to calculate the analytical vibration frequency in vacuum with the following expression:

$\omega_{v a c}=\sqrt{\frac{C_{n}^{4} E I}{L^{4} \rho}}$

where the subscript $n$ denotes the mode and $C_{n}$ and the positive roots of equation (43)

$1+\cos \left(C_{n}\right) \cosh \left(C_{n}\right)=0$.

Taking the first vibration mode, $C_{1}=1.8751$ and the frequency of the beam becomes:

$\omega_{v a c}=\sqrt{\frac{C_{1}^{4} E I}{L^{4} \rho}}=32.097 \mathrm{rad} / \mathrm{s} \rightarrow 5.108 \mathrm{~Hz}$

The frequency observed in the simulation for low fluid densities $\left(<0.001 \mathrm{~kg} / \mathrm{m}^{3}\right)$ is $5.175 \mathrm{~Hz}$, in good agreement with the theoretical value. The slightly higher value is caused by the low number of elements in the thickness, which leads to a stiffer behavior.

To test the influence of the fluid density, according to Van Eysden [21] it is possible to estimate the lower vibration modes for rectangular section beams immersed in a fluid using the following formula:

$\omega_{\text {fluid }}=\omega_{\text {vac }}\left(1+\frac{\pi \rho_{p} b}{4 \rho_{q} H} \Gamma_{f}\right)^{-1 / 2}$

where $\Gamma_{f}$ is the hydrodynamic function which depends on the viscosity and the cross section of the beam. For inviscid flows and square sections $\Gamma_{f}=1.513$ [3].

Fluid densities from $0.0001 \mathrm{~kg} / \mathrm{m}^{3}$ to $0.99 \mathrm{~kg} / \mathrm{m}^{3}$ were selected to validate the code. The results can be seen in Figure 18, toghether with the comparison against (45). Analyzing this graph, it is clear that the numerical solution diverges from the expected curve as the fluid density increases. This is caused by the limitations of the standard linear elements used for the velocity field, which lead to interface elements that are assembled with averaged (based on volumes) stiffness properties between the fluid and the solid and therefore the added mass effect is larger than it should be.

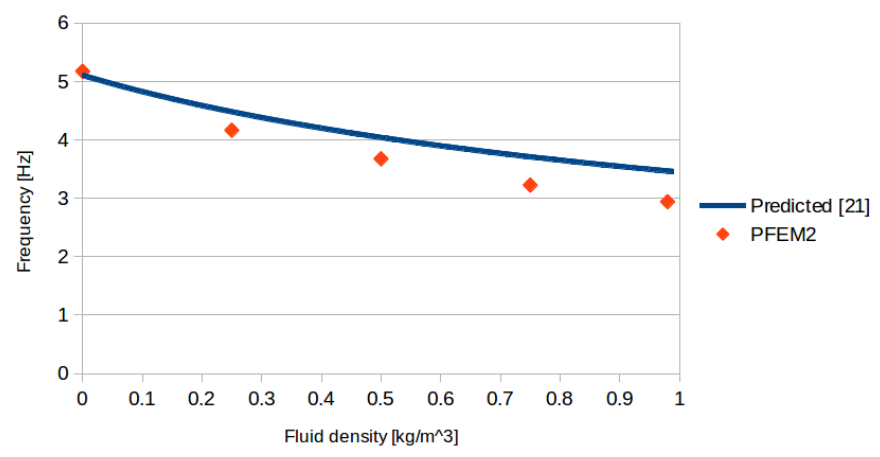

Fig. 18: Oscillation frequency of the 3D cantillever beam for different fluid densities

To analyze the convergence of the method with mesh size, the case with a fluid density $\rho_{f}=0.5 \mathrm{~kg} / \mathrm{m}^{3}$ was selected. Mesh sizes from $h=0.33$ to $h=0.08$ were used ( 3 to 12 elements in the thickness of the beam). In all the cases the interface was located splitting the elements in the middle to test the worst case scenario.

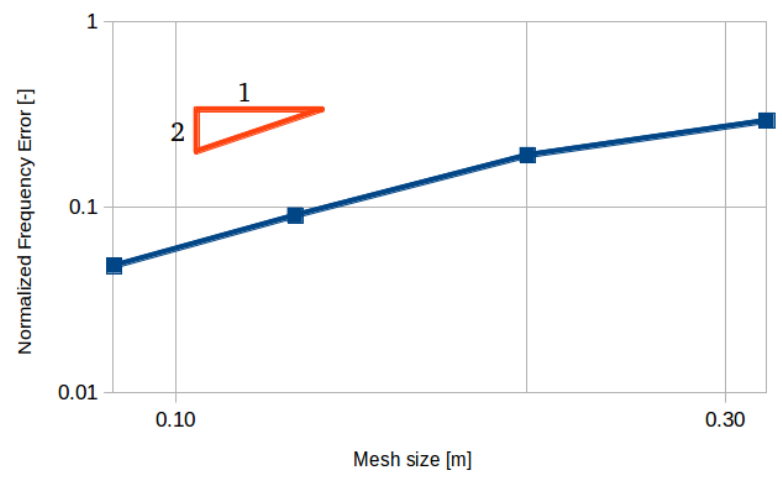

Fig. 19: Mesh convergence for the oscillation frequency of the $3 \mathrm{D}$ cantilever beam

Quadratic convergence on mesh size can be observed in Figure 19, indicating that the interaction between the fluid and the beam is accurately simulated.

\section{Conclusions and future work}

A tool to solve monolithically multi-fluids and FSI problems was developed. The strategy is based on the PFEM2 originally formulated in [14] for homogeneous fluids, which allows for fast computations while maintaining accuracy. 
The developed model makes use of Lagrangian particles and a fixed mesh. In this mesh, several enrichment degrees of freedom (DoFs) are added to improve the pressure field at the interfaces. Adding extra DoFs allows for a more accurate capturing of the solution where the material properties change abruptly since this leads to discontinuities of the pressure or its gradients. On the other hand, to avoid resizing the system of equations, the new variables are statically condensed and therefore computation times are not significantly increased.

The last example shows that the standard velocity field might not be accurate for coarse meshes due to the artificial added mass effect caused by the FE linear shape functions. As a next step, enrichening the velocity variables to improve the definition of the interface will reduce errors without the need of refining the mesh.

Finally, a major improvement in the capabilities of the solver will be adding more advanced constitutive models to account for a more realistic behavior of complex phenomena such as debris flows, both at the solid stage and the rheological model of the flow. This will allow for the simulation of the complete phenomena, from initiation to deposition of the eroded material using a single and accurate tool. Currently, there are almost no strategies which can simulate the whole process using a unified frame.

\section{Acknowledgements}

The research leading to these results has received funding from the People Programme (Marie Curie Actions) of the European Union's Seventh Framework Programme FP7/2007-2013/ under REA grant agreement n 289911. This work was also supported by the ERC Advanced Grant REALTIME, project AdG-2009325, by the ERC Advance Grant SAFECON, project AdG-26752 and by the HFLUIDS project of the National RTD Plan of the Spanish Ministry of Science and Innovation I+D BIA2010-15880.

\section{References}

1. Belytschko, T., Liu, W.K., Moran, B., Elkhodary, K.: Nonlinear finite elements for continua and structures. John Wiley \& Sons (2013)

2. Bochev, P.B., Gunzburger, M.D., Lehoucq, R.B.: On stabilized finite element methods for the stokes problem in the small time step limit. International Journal for Numerical Methods in Fluids 53(4), 573-597 (2007)

3. Brumley, D.R., Willcox, M., Sader, J.E.: Oscillation of cylinders of rectangular cross section immersed in fluid. Physics of Fluids (1994-present) 22(5), 052,001 (2010)

4. Codina, R.: Stabilization of incompressibility and convection through orthogonal sub-scales in finite element methods. Computer methods in applied mechanics and engineering 190(13), 15791599 (2000)

5. Codina, R.: Stabilized finite element approximation of transient incompressible flows using orthogonal subscales. Computer Methods in Applied Mechanics and Engineering 191(39), 4295-4321 (2002)

6. Codina, R., Badia, S.: On some pressure segregation methods of fractional-step type for the finite element approximation of incompressible flow problems. Computer Methods in Applied Mechanics and Engineering 195(23), 2900-2918 (2006)

7. Coppola, H.: A finite element model for free surface and two fluid flows on fixed meshes. Ph.D. thesis, Universitat Politècnica de Catalunya (2009)

8. Dadvand, P., Rossi, R., Oñate, E.: An objectoriented environment for developing finite element codes for multi-disciplinary applications. Archives of computational methods in engineering $\mathbf{1 7}(3)$, 253-297 (2010)

9. DeBlois, B.M.: Linearizing convection terms in the navier-stokes equations. Computer methods in applied mechanics and engineering 143(3), 289-297 (1997)

10. Felippa, C.A.: Introduction to finite element methods. University of Colorado, Boulder, http://www. colorado. edu/engineering/CAS/courses. d/IFEM. d (2004)

11. Fries, T.P., Matthies, H.G.: A review of petrovgalerkin stabilization approaches and an extension to meshfree methods. Institute of Scientific Computing, Univ. of Braunschweig-Inst. of Technology, TR-2004-01, Brunswick, Germany (2004)

12. Guermond, J.L., Quartapelle, L.: A projection fem for variable density incompressible flows. Journal of Computational Physics 165(1), 167-188 (2000)

13. He, X., Chen, S., Zhang, R.: A lattice boltzmann scheme for incompressible multiphase flow and its application in simulation of rayleigh-taylor instability. Journal of Computational Physics 152(2), 642-663 (1999)

14. Idelsohn, S., Nigro, N., Gimenez, J., Rossi, R., Marti, J.: A fast and accurate method to solve the incompressible navier-stokes equations. Engineering Computations 30(2), 2-2 (2013)

15. Idelsohn, S.R., Marti, J., Becker, P., Oñate, E.: Analysis of multifluid flows with large time steps using the particle finite element method. International Journal for Numerical Methods in Fluids 
(2014)

16. Idelsohn, S.R., Marti, J., Limache, A., Oñate, E.: Unified lagrangian formulation for elastic solids and incompressible fluids: application to fluid-structure interaction problems via the pfem. Computer Methods in Applied Mechanics and Engineering 197(19), 1762-1776 (2008)

17. Muneer, T.T., Kubie, J., Grassie, T.: Heat transfer: a problem solving approach. Taylor \& Francis (2003)

18. Oñate, E., Franci, A., Carbonell, J.M.: A particle finite element method for analysis of industrial forming processes. Computational Mechanics pp. 1-23 (2014)

19. Oñate, E., Franci, A., Carbonell, J.M.: A particle finite element method (pfem) for coupled thermal analysis of quasi and fully incompressible flows and fluid-structure interaction problems pp. 129156 (2014)

20. Turek, S., Hron, J.: Proposal for numerical benchmarking of fluid-structure interaction between an elastic object and laminar incompressible flow. Springer (2006)

21. Van Eysden, C.A., Sader, J.E.: Resonant frequencies of a rectangular cantilever beam immersed in a fluid. Journal of applied physics 100(11), 114,916 (2006)

22. Wendland, H.: Piecewise polynomial, positive definite and compactly supported radial functions of minimal degree. Advances in computational Mathematics 4(1), 389-396 (1995)

23. Zienkiewicz, O., Taylor, R.: The Finite Element Method: The Basis, vol. 1. Butterworth-heinemann (2000)

24. Zienkiewicz, O., Taylor, R., Zhu, J.Z.: The Finite Element Method: Its Basic and Fundamentals, vol. 1, 6th edn. Elsevier (2006) 